\title{
Static Metrological Characterization of a Ferrimagnetic Resonance Transducer for Real-Time Magnetic Field Markers in Particle Accelerators
}

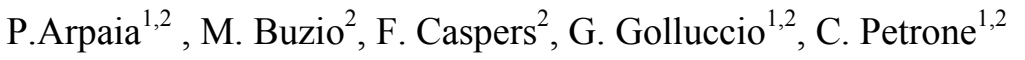 \\ 1) Department of Engineering, University of Sannio, \\ Corso Garibaldi 107, 82100 Benevento, Italy. Ph : +39 \\ 0824305804-17, Fax: +39 0824305840 , \\ E-mail: arpaia@unisannio.it \\ 2) CERN, European Organization for Nuclear Research, \\ 1211 Geneva 23, Switzerland. Ph: +41 227676111 , \\ E-mail: giancarlo.golluccio@,cern.ch
}

\begin{abstract}
The metrological characterization in a static magnetic field of a transducer based on ferrimagnetic resonance for realtime markers in particle accelerators is reported. The transducer is designed to measure the magnetic field with a precision of $10 \mu \mathrm{T}$. A case study on the new real-time field monitoring system for the CERN accelerators highlighting the performance of the new ferrimagnetic transducer is described. Preliminary experimental results of the characterization for static fields are discussed.
\end{abstract}

Keywords - Particle accelerators, magnetic field measurements, field marker, Ferrimagnetic materials, Magnetometers.

\section{INTRODUCTION}

In particle accelerators, the real-time knowledge of the magnetic field used to bend and focus the beam is essential for the operation. Several subsystems such as power supplies, RF cavities, and beam control use this information as feedback in the control loop. Typically, real-time monitoring is obtained by means of high-performance magnetic measurement systems [1] or mathematical models of the magnet response [2]. The measurements are carried out usually in a reference magnet powered in series with the accelerator magnets chain. Data are then distributed to the different subsystems, typically as two parallel incremental and decremental digital pulse trains ("Btrains"). By simply counting the pulses, each user can reconstruct the magnetic field as a function of the time. The demanding requirements in terms of accuracy, time response, and precision, are satisfied practically fully by the coil fluxmeter as a field instrument. However, an additional measurement is needed to retrieve a field value to be used as integration constant. In most cases, a measurement system able to provide a digital trigger pulse as the field crosses a given threshold ("field marker") is used.

The peaking strip is used as marker since the commissioning of the CERN Proton Synchrotron (PS) [3]. Such a transducer is composed by a thin strip of material with high permeability and an almost rectangular $B-H$ cycle [4]. The strip is immersed in a bias field, equal and opposite to the field to mark, generated by an excitation coil. A strong voltage pulse is generated in a detection coil when the external field $H$ crosses the threshold value, thus causing the magnetization to flip. Reference [4] describes the performance and limitations of this transducer. The achievable accuracy is mainly dominated by the width of the pulse. The uncertainty limit is fixed to $\pm 2 \mu \mathrm{T}$ for $\mathrm{a} \mathrm{dB} / \mathrm{dt}$ of $1 \mathrm{~T} \mathrm{~s}^{-1}$. Eddy currents in the strip can decrease the total changing flux in the coil. The main advantage of this transducer is the compatibility with field gradients, improved by reducing its size. However, the complexity of construction and the need for very large bias coils limit their use at high fields.

For the control system of the accelerator facilities of the Japanese high-energy research organization (KEK) [5], and for the B-Train of CNAO (Centro Nazionale Adroterapia Oncologica) [6], a Hall probe is used as field marker to measure the field at the bottom of the cycle. This system can achieve a good accuracy by a suitable temperature drift correction and a periodic calibration of the sensor.

Different studies $[5,7,8]$ were carried out to explore the use Nuclear Magnetic Resonance (NMR) probes as field marker. Transducers based on NMR are currently the magnetic measurement systems with the best absolute accuracy in a large field range [9]. NMR working principle is related to the precession frequency of the proton spin in a hydrogen nucleus with an external magnetic field by means of a gyro-magnetic ratio. It can be adopted (i) as field marker in a changing field, once the NMR probe was excited at a given frequency by an RF oscillator, or (ii) as a teslameter, by sweeping in frequency and locking on the energy absorption resonance. Such systems are used widely in industry for their low uncertainty of a few of ppm [10]. Their performance degrades in inhomogeneous fields because the spin in all regions of the probe does not respond at the same frequency. In such a case, the system cannot lock on the absorption frequency when the field gradient is important (usually higher $200 \mathrm{ppm} / \mathrm{cm}$ for commercial NMR). Better performance is obtained by compensating the field gradient with coil or homogenizing plates [11].

In this paper the static metrological characterization of an alternative solution, based on the Ferrimagnetic Resonance effect (FMR) [12], is presented for the problem of marking the field in particle accelerator magnets. The FMR exploits an 
electron spin resonance in ferrimagnetic material. The gyromagnetic ratio of FMR materials such as Yttrium Iron Garnet (YIG) is about $28 \mathrm{GHz} / \mathrm{T}[13,14]$, and for this reason they are commonly used in microwave equipment as tunable filters [15]. The high Q factor allows small garnets insensitive to field gradients. This study, carried out at CERN in collaboration with the University of Sannio, is concerned with the evaluation of the FMR performance with the final aim of showing its suitability as a sensor for new real-time monitoring system for CERN accelerators.

\section{REQUIREMENTS}

At CERN facilities, different accelerators use B-train for measuring in real-time magnetic field during operation [11]. In Fig. 1, the principle of the B-Train of PS is shown. A peaking strip used as a field marker starts the digital train. The frequency of the pulses is proportional to the field variation probed by a fixed coil with an equivalent surface $A_{\text {coil }}$ during the ramp-up and ramp-down of the magnet. The coil voltage is proportional to the field time derivative, thus the field measured is:

$$
B(t)=B_{\text {marker }}\left(t_{1}\right)+\int_{t_{1}}^{t} \dot{B} d t
$$

where $B_{\text {marker }}\left(t_{1}\right)$ is the field measured by the marker at about $5 \mathrm{mT}$ (below the injection flat bottom).

A second marker in the field flat top or in the higher part of the ramp is desirable to calculate in situ the systematic calibration error $\Delta$ of the coil:

$$
\Delta=\frac{\int_{t_{1}}^{t_{2}} \dot{B} d t}{B_{\text {marker }}\left(t_{2}\right)-B_{\text {marker }}\left(t_{1}\right)}-1
$$

The most demanding application is the PS, providing proton beams of $26 \mathrm{GeV}$ to the Large Hadron Collider (LHC) [7]. The main magnets combine functions of dipole and quadrupole fields for bending and focusing the beam, respectively, with a field gradient up to $4 \% / \mathrm{cm}$. In addition, the field ramp rates reach up to $2.3 \mathrm{~T} / \mathrm{s}$, with a flat top value ranging from few tenths to about $1.2 \mathrm{~T}$ according to the PS operation cycle. In Fig. 2, the present real-time measurement system of the PS is sketched. Several coils placed in different positions of the reference magnet aperture provide the $\dot{B}$ signal after a suitable amplification. The generated digital train pulse has a resolution of $10 \mu \mathrm{T}$ : at each field increment of $10 \mu \mathrm{T}$, a pulse is released to the different users through a digital control card.

Future requirements in terms of beam quality for the LHC have a direct impact on the performance, first of the PS in general, and, more specifically, on the field measurement. The challenge of the new system is to achieve $\pm 5 \mu \mathrm{T}$ of precision on the digital train pulse. In addition, the field marker has to be able to work in a range from 60 to $500 \mathrm{mT}$ and to preserve long-term stability (at most $\pm 50 \mu \mathrm{T} /$ year).

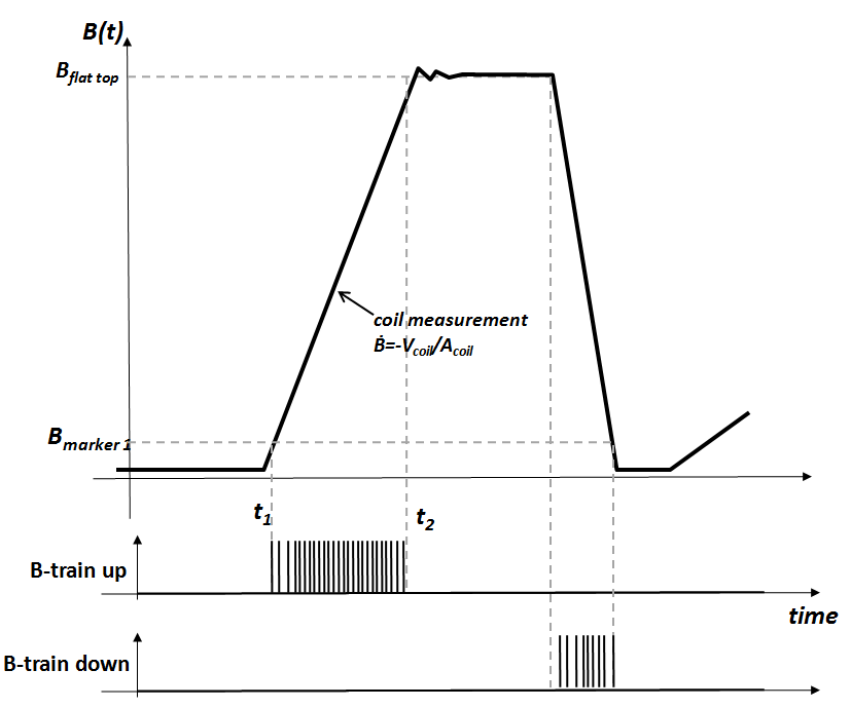

Figure 1: Principle of B-train system.

\section{FMR FOR FIELD MARKER}

The research is devoted to show the applicability of a single-crystal ferrimagnetic resonance transducer as field marker for the measurement of the reference field of the PS magnets. In [15], details about (i) the response of this class of filters in terms of resolution related to the Q-Factor, and (ii) the capability of a YIG filter to maintain high performance in non-homogeneous fields, are given.

Single crystal YIG filters can reach high $Q$ values $(\sim 10,000)$ but their response to external fields depends on the temperature and on the alignment of the YIG sphere in the resonator [17]. Polycrystalline spheres can achieve better performance also, namely a flat response (less than $10^{-}$ 5 /degree) in the temperature range of interest from 25 to $55^{\circ} \mathrm{C}$, although with a $\mathrm{Q}$ about ten times lower. YIG filters have to work in the saturation region of the ferrimagnetic material to ensure homogeneous magnetization and a $\mathrm{Q}$ as high as possible, thus constraining the lower limit to about $0.06 \mathrm{~T}$. The upper limit imposed by current microwave technology is about $3 \mathrm{~T}$, corresponding to $80 \mathrm{GHz}$. A YIG filter can be used essentially in two configurations to measure a field: (i) as a teslameter in a static field, by sweeping in frequency the filter input signal and detecting the maximum of energy absorption and (ii) as a marker in a time-varying field, by measuring the interval time between the appearance of the resonance and an external time reference, such as the start trigger of a current ramp. Owing to the small dimension of the sensing element (typical diameter of $0.3 \mathrm{~mm}$ ), relatively high field gradients can be tolerated without a severe degradation of the Q-Factor. This behavior makes such a device a good field marker compared to: i) NMR probes, because no compensation of the gradient is needed, ii) the peaking strips, because a large range of fields can be measured without external bias coils. 


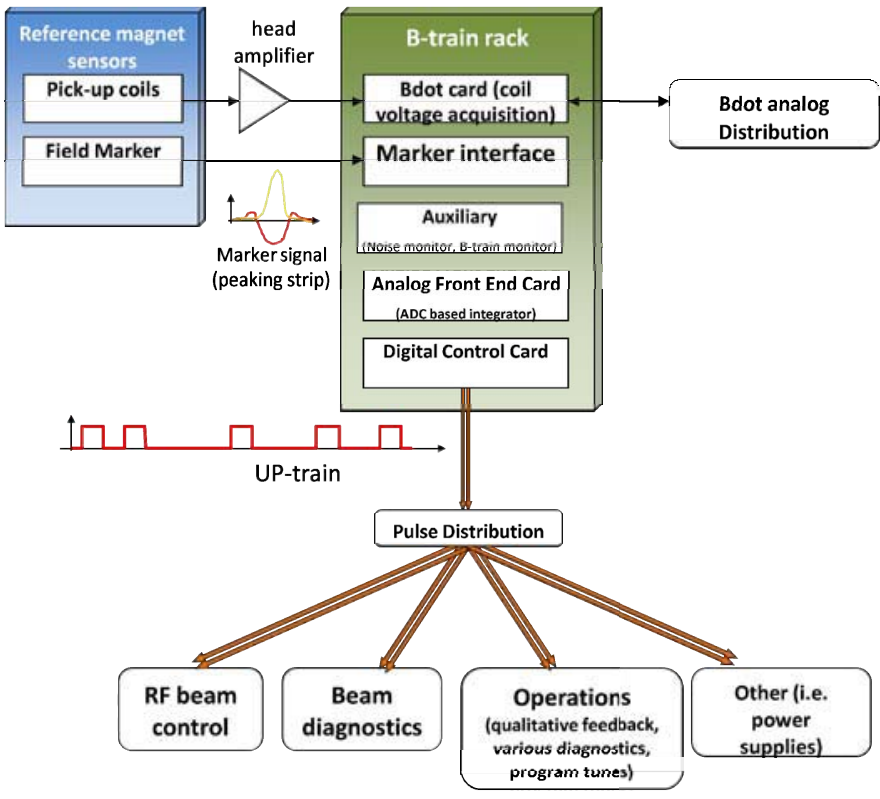

Figure 2: Overview of the "B-Train" system of PS.

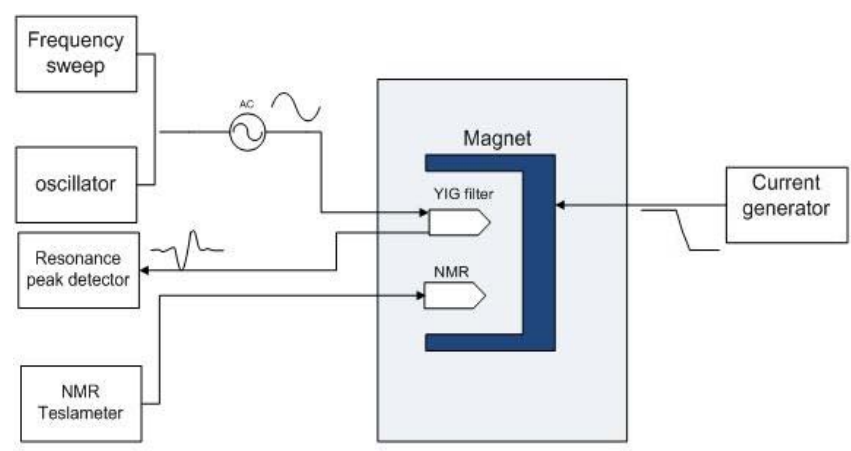

Figure 3: Setup of the FMR field marker.

\section{EXPERIMENTAL RESULTS}

In this Section, i) the experimental setup for the metrological characterization campaign of the ferrimagnetic resonance transducer for field marker use and ii) the first experimental results in static condition are reported.

\section{Experimental Setup}

In Fig. 3, the measurement setup for demonstrating experimentally the suitability of FMR as field marker is illustrated. A Agilent ${ }^{\circledR}$ microwave Network Analyzer is used both as a signal generator and a resonance detector, working in the range from 1.6 to $9.0 \mathrm{GHz}$ corresponding to about 60 to $320 \mathrm{mT}$. The device is set to measure the transmission coefficient $S_{21}$ of the network for each frequency starting from $1.6 \mathrm{GHz}$. In this range, the YIG filter (built for this purpose by Omniyig $^{\circledR}$ ) has a Q-factor of about 1,000 . The magnetic field is generated by a reference dipole magnet with a transfer function of $315 \mathrm{mT} / \mathrm{A}$, previously mapped by means of a NMR probe with an absolute uncertainty of $\pm 5 \mathrm{ppm}$ [10]. Two NMR probes, covering the field range under test as a whole, are also installed close to the FMR in turn, in order to provide a reference. To avoid any RF interference between the NMR and FMR systems, only one is activated at a time. In Fig. 4, the field map of the magnet along its length as a whole at four different lateral positions is shown. The horizontal variation is less than $1 \cdot \mu \mathrm{T} / \mathrm{mm}$ (between $\pm 30 \mathrm{~mm}$ ), while, in the central region between 1120 and $1200 \mathrm{~mm}$, where FMR transducer and the NMR probe are placed, the longitudinal field gradient is around $5 \cdot \mu \mathrm{T} / \mathrm{mm}$, assuring a displacement between the probes of a few of millimeters not influencing the results. A current generator with high stability and low ripple (10 ppm relative to the nominal value) is used to excite the magnet. This can generate a maximum current of $40 \mathrm{~A}$ at $8 \mathrm{~V}$, thus fixing the upper limit of investigation to about $0.135 \mathrm{~T}$. All the measurements were carried out on a stable hysteresis cycle of the magnet in order to compare the results obtained over several current cycles. The stabilization is obtained simply by 5 pre-cycles of the magnet. The peak frequency measurement is compared to the value obtained with the NMR PT2025 teslameter once the field is stabilized for the static characterization.

\section{Experimental results}

The resonance frequency of the YIG filter is evaluated by the peak detection of $S_{2 l}$ provided directly by the network analyzer. The corresponding error is assessed by comparing the resonance curves measured by the network analyzer with a parabolic fitting. The high SNR of the transducer guarantees the uncertainty of the resonance peak detection to be less than $\pm 0.005 \mathrm{~dB}$. In other words, the difference between the resonance frequency detected by the network analyzer and the one defined by the parabola maximum is less than $10 \mathrm{kHz}$, corresponding to $10^{-8} \mathrm{~T}$. In Figs. 5 and 6 , the results of the static calibration of the FMR transducer with respect to the NMR are reported as deterministic error and $3-\sigma$ relative uncertainty band respectively. The gyro-magnetic ratio calculated is $28.084 \mathrm{GHz} / \mathrm{T}$, the theoretical value of $28 \mathrm{GHz} / \mathrm{T}$ is degraded by material impurity [18]. An offset of $10 \cdot \mu \mathrm{T}$ in the static calibration agrees with a displacement of $2 \mathrm{~mm}$ between the YIG filter and the NMR reference probe, according to the non-homogeneity of the field shown in Fig. 4. The deterministic error trend reveals also a parabolic nonlinearity of about $0.06 \mathrm{mT}$ in the full range of investigation. This phenomenon, to be investigated, may be due to the incomplete saturation of the material. This nonlinearity is

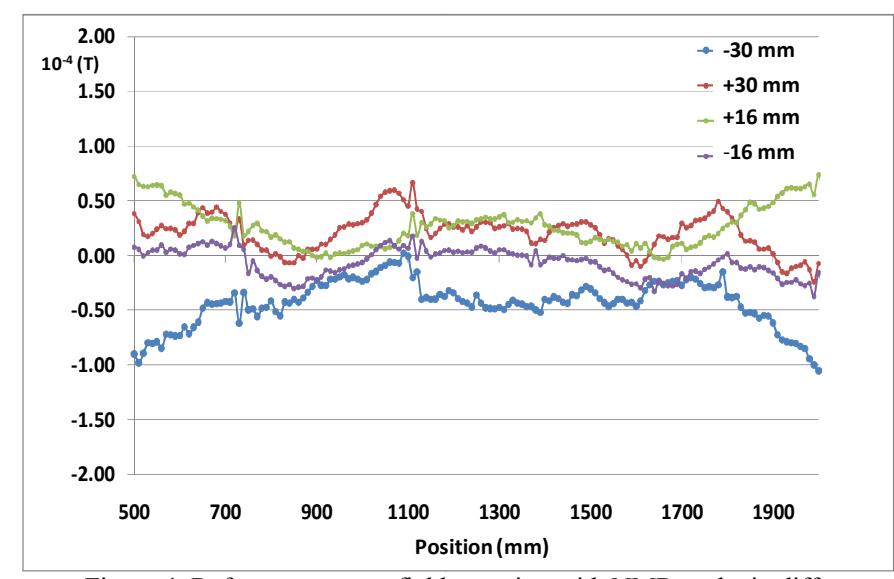

Figure 4: Reference magnet field mapping with NMR probe in different horizontal positions with respect to the centre of the magnet (central field $1 \mathrm{~T}$ ). 


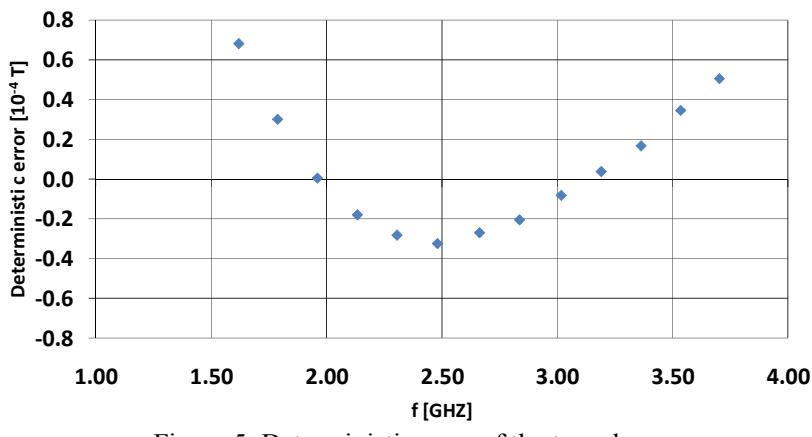

Figure 5: Deterministic error of the transducer.

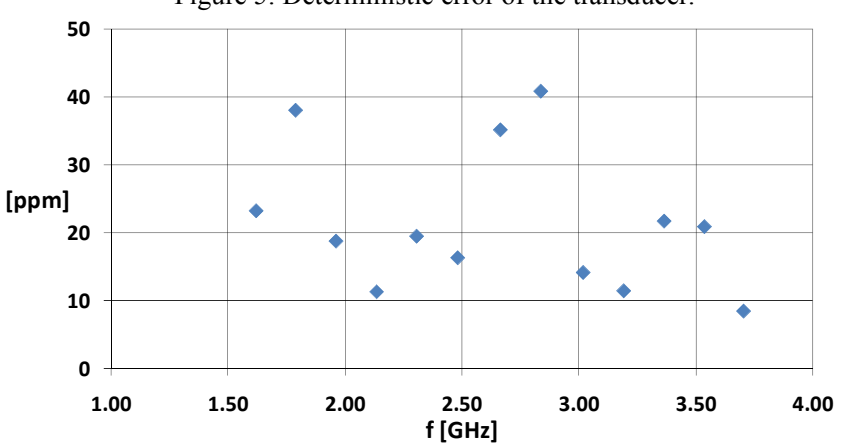

Figure 6: 3- $\sigma$ uncertainty of the transducer.

measured to be very stable over several days. The relative uncertainty (Fig.6) has a maximum of \pm 40 ppm over the full range, confirming the suitability of the FMR transducer $( \pm$ $2 \mu \mathrm{T}$ absolute uncertainty) as field marker. The high static performance has to be confirmed with a calibration in dynamic conditions. Therefore, the system not only matches the requirements of the real-time measurement system for the PS accelerator, but could also be considered as a basis for a general-purpose teslameter.

\section{CONCLUSION}

The metrological performance of a ferrimagnetic resonator to be used as field marker for a real-time field measurement system is presented. The calibration in a static field of the transducer shows $\pm 40 \mathrm{ppm}$ uncertainty up to $0.13 \mathrm{~T}$, well suitable for the present application. Nonlinearity in the response of $0.05 \mathrm{mT}$ for fields below $0.08 \mathrm{~T}$ has to be investigated and corrected to improve the accuracy of the final system. Future work will test the transducer in dynamic conditions, i.e. in field ramps up to $2 \mathrm{~T} / \mathrm{s}$.

\section{ACKNOWLEDGMENTS}

Authors thank L. Walckiers for his useful suggestions, W. Capogeannis from Omniyig for producing the new FMR sensor according to CERN specifications, J.P. Garcia, P. Galbraith and D. Cote for their contribution to the setup of the tests.

\section{REFERENCES}

[1] P. D. Eversheim, P. Von Rossen, B. Scholler and F. Hinterberger "A fast Precise Magnetic Field Control",Nuclear Instruments and Methods, Vol. 160, 1979 pp. 345-351.

[2] N. Sammut, L . Bottura, J. Micallef "Mathematical formulation to predict the harmonics of the superconducting Large Hadron Collider magnets" Physical Review Special Topic Accelerators and Beams, Vol. 9, 2006.

[3] J. L. Symods "Methods of Measuring Strong Magnetic Fields" Reports on Progress in Physics, Vol. 18, 1955.

[4] J. M. Kelly, "Magnetic Field Measurements with Peaking Strips", Review of Scientific Instruments, Vol. 22, pp. 256-258, 1957.

[5] T. Kasuga "B-clock system for KEK main ring" IEEE Transaction on Nuclear Science, Vol. NS-24, No. 3, pp. 1742-1744, 1977.

[6] G. Franzini,et al. "Final Design and Features of the B-Train System of CNAO", Proceedings of IPAC '10, Kyoto Japan.

[7] F.Caspers "The ferrimagnetic resonance marker (FMR) in the PS", International Magnetic Measurement Workshop (IMMW10), Batavia, Illinois, 1997.

[8] D.Cornuet "Search for an industrial field marker inside the PS reference magnet", International Magnetic Measurement Workshop (IMMW13), Stanford, California, 2003.

[9] K. N. Henrichsen, „Magnetic field imaging” in „Encyclopedia of imaging science and technology" V. J. P. Hornak, pp. 970-977, Wiley-VCH Verlag, New York, NY, USA, 2002.

[10] P. Sommer,"The Metrolab PT2026. A new generation of teslameter", International Magnetic Measurement Workshop (IMMW13), Stanford, California, 2003.

[11] A. Beaumont, M. Buzio,"A NMR marker for CERN PS CombinedFunction Magnet", International Magnetic Measurement Workshop (IMMW16), Bad Zurzach, Switzerland,2009.

[12] S.V. Vonsovskii, "Ferrimagnetic Resonance", Pergamon Press, Oxford,1966, pp. 24-30.

[13] N. Kernevez, D. Duret, M. Moussavi, J.M. Leger, "Weak field NMR and ESR spectrometers and magnetometers", IEEE Trans. on Magn., 28 (5), (1992) 3054-3059.

[14] H. K. Carpenter, M. K. daSilva "Phase-locked yttrium iron garnet magnetometer for remote measurement of small field changes in a fluctuating background", Rev. Sci. Instrum., vol. 53, pp. 1414 - 1417.

[15] P.Roschomann, "Compact YIG bandpass Filter with Finite-Pole frequencies for Applications in Microawve Integrated Circuit", IEEE transaction on Microwave theory an techniques, 1973.

[16] M.Benedikt, F.Caspers, M.Lindroos“Application of Magnetic Markers for Precise Measurement of Magnetic Fields in Ramped Accelerators" Particle. Accelerators.: 63 (1999)

[17] F.K. Beckmann, H. Dötsch, P. Röschmann and W. Schilz, "Remote Temperature Sensing in Organic Tissue by Ferrimagnetic Resonance Frequency Measurements",11th European Microwave Conference, Amsterdam, 1981.

[18] N. Vukadinovic, J. Ben Youssef b, H. Le Gall "Influence of magnetic parameters on microwave absorption of domain mode ferromagnetic resonance.", Journal of Magnetism and Magnetic Materials, Vol. 150, pp. 213-222, 1995. 